\title{
High velocity and hypervelocity stars from the companions of type Ia supernovae
}

\author{
B. Wang, D. Liu, S. Jia and Z. Han \\ Key Laboratory for the Structure and Evolution of Celestial Objects, Yunnan Astronomical \\ Observatory, CAS, Kunming, 650011, China (email: wangbo@ynao.ac.cn)

\begin{abstract}
The single-degenerate (SD) model is currently a favourable progenitor scenario for the production of type Ia supernovae (SNe Ia). The companion in the SD model would survive after the SN explosion. In this work, we obtained the velocity distribution of the surviving companion stars from different SD scenarios in the Galaxy, which can be verified by future observations.
\end{abstract}

Keywords. binaries: close - supernovae: general - white dwarfs

\section{Introduction}

Type Ia supernovae (SNe Ia) are crucial for the studies of stellar evolution, galaxy evolution and cosmology. They are generally thought to be thermonuclear explosions of accreting carbon-oxygen white dwarfs (CO WDs) in close binaries, however, the nature of the mass donor star is still unclear (e.g., Wang \& Han 2012). There are mainly two progenitor scenarios, i.e., the single-degenerate (SD) and double degenerate (DD) models. The companion in the SD model would survive after the SN explosion and potentially be identifiable, whereas an explosion following the merger of two WDs would leave no remnant (e.g., Han 2008; Wang \& Han 2010). Thus, one way to distinguish between the $\mathrm{SD}$ and DD models is to search surviving companion stars of SNe Ia.

\section{Model and Results}

We obtained the tangential and radial velocity distribution of the surviving companion stars from different SD models in the Galaxy (including the WD + MS scenario and the WD + He star scenario), which can be verified by future observations (for details see Jia et al. 2013). The surviving companion stars from the WD + MS scenario could potentially explain the properties of high velocity stars in the Galaxy, whereas the surviving companion stars from the WD + He star scenario have a high spatial velocity $(¿ 400 \mathrm{~km} / \mathrm{s})$, which could be an alternative origin for hypervelocity stars (HVSs), especially for HVSs such as US 708 (see also Wang \& Han 2009).

\section{References}

Han, Z. 2008, ApJ, 677, L109

Jia, S., Wang, B., \& Han, Z. 2013, A\&A, in prep

Wang, B., \& Han, Z. 2009, A\&A, 508, L27

Wang, B., \& Han, Z. 2010, MNRAS, 404, L84

Wang, B., \& Han, Z. 2012, New Astron. Rev., 56, 122 